\title{
Fabrication and Characterization of a Micromechanical Sensor for Differential Detection of Nanoscale Motions
}

\author{
Cagri A. Savran, Andrew W. Sparks, Joachim Sihler, Jian Li, Wan-Chen Wu, Dean E. Berlin, \\ Thomas P. Burg, Student Member, IEEE, Jürgen Fritz, Martin A. Schmidt, Senior Member, IEEE, and Scott R. Manalis
}

\begin{abstract}
We have micromachined a mechanical sensor that uses interferometry to detect the differential and absolute deflections of two adjacent cantilevers. The overall geometry of the device allows simple fluidic delivery to each cantilever to immobilize molecules for biological and chemical detection. We show that differential sensing is 50 times less affected by ambient temperature changes than the absolute, thus enabling a more reliable differentiation between specific cantilever bending and background effects. We describe the fabrication process and show results related to the dynamic characterization of the device as a differential sensor. The root-mean-squared (rms) sensor noise in water and air is $\sim 1 \mathbf{~ n m}$ over the frequency range of $0.4-40 \mathrm{~Hz}$. We also find that in air, the deflection resolution is limited only by the cantilever's thermomechanical noise level of $0.008 \AA / \mathrm{Hz}^{1 / 2}$ over the frequency range of 40-1000 Hz.

[781]

Index Terms-AFM technology, BioMEMS, biosensor, chemical-biological functionalization, diffraction, interdigitated, silicon nitride.
\end{abstract}

\section{INTRODUCTION}

$\mathbf{T}$ HE use of micromachined cantilevers for detection of chemical and biological interactions of molecules has been investigated in recent years. It has been shown that intermolecular forces resulting from binding of molecules can bend a flexible micromachined cantilever [1]-[6].

Manuscript received November 21, 2001; revised May 24, 2002. This work was supported by the Defense Advanced Research Projects Agency (DARPA) Bio-Info-Micro (BIM) Program, Air Force Office of Scientific Research (AFOSR), Department of Defense (DOD), Motorola, and the MIT Media Laboratory Things That Think (TTT) consortium. Subject Editor C.-J. Kim.

C. A. Savran is with the Media Laboratory and the Department of Mechanical Engineering, Massachusetts Institute of Technology, Cambridge, MA 02139 USA.

A. W. Sparks is with the Media Laboratory and the Department of Materials Science and Engineering, Massachusetts Institute of Technology, Cambridge, MA 02139 USA.

T. P. Burg is with the Media Laboratory and the Department of Electrical Engineering and Computer Science, Massachusetts Institute of Technology, Cambridge, MA 02139 USA

J. Fritz is with the Media Laboratory, Massachusetts Institute of Technology, Cambridge, MA 02139 USA

J. Sihler, J. Li, and W.-C. Wu are with the Department of Mechanical Engineering, Massachusetts Institute of Technology, Cambridge, MA 02139 USA.

D. E. Berlin is with the Department of Materials Science and Engineering, Massachusetts Institute of Technology, Cambridge, MA 02139 USA.

M. A. Schmidt is with the Department of Electrical Engineering and Computer Science and the Microsystems Technology Laboratories, Massachusetts Institute of Technology, Cambridge, MA 02139 USA.

S. R. Manalis is with the Media Laboratory and the Division of Biological Engineering, Massachusetts Institute of Technology, Cambridge, MA 02139 USA (e-mail: scottm@media.mit.edu).

Digital Object Identifier 10.1109/JMEMS.2002.805057
Fritz et al. used two adjacent micromachined cantilevers to detect DNA hybridization [7]. Commercially available pipettes were used to functionalize the surfaces of the cantilevers with DNA oligonucleotides of different sequences. DNA hybridization on one of the cantilevers and not on the other caused a differential bending, i.e., the bending of one cantilever relative to the other. The bending was determined by the optical lever method whereby a laser beam was focused on the terminus of each cantilever and the location of the reflected beam was measured with a position-sensitive photodetector. An important result of this study was that taking the difference of the deflection signals from the two identical cantilevers significantly reduced the effect of temperature and optical index changes that occur in solution. As a result, only the bending induced by the biomolecular interactions of the molecules was revealed in the differential signal.

In bending experiments with single cantilevers [1]-[6], [12], the ambient temperature has to be controlled precisely during the experiment to avoid bending due to the temperature sensitivity of the cantilevers. In addition, controls to determine the specificity of the bending have to be done sequentially using different cantilevers. This can introduce additional uncertainties due to variations in experimental conditions.

In this paper, we present a sensor that inherently detects the differential bending of two adjacent cantilevers. This eliminates the need for electronic subtraction of the signals downstream, and also relaxes the alignment tolerances of the components used in the optical lever method. In order to achieve direct mechanical subtraction of the deflection signals, we used interdigitated (ID) fingers that formed a diffraction grating between the two adjacent cantilevers. The sensor also has ID fingers that enable detecting the absolute bending of each cantilever. Manalis et al. introduced the use of ID fingers for atomic force microscopy and showed that subangstrom level resolution can be achieved [8]. Here, we describe how this concept is incorporated with a device geometry that allows fluidic delivery to each cantilever surface using commercially available pipettes. We also characterize the device as a differential sensor in terms of similarity of the adjacent cantilevers, the response to background noise, and the deflection resolution.

\section{DeVICE Design AND FABRICATION}

A schematic of the device illustrating the optical detection and actuation is shown in Fig. 1(a). The device consists of two 


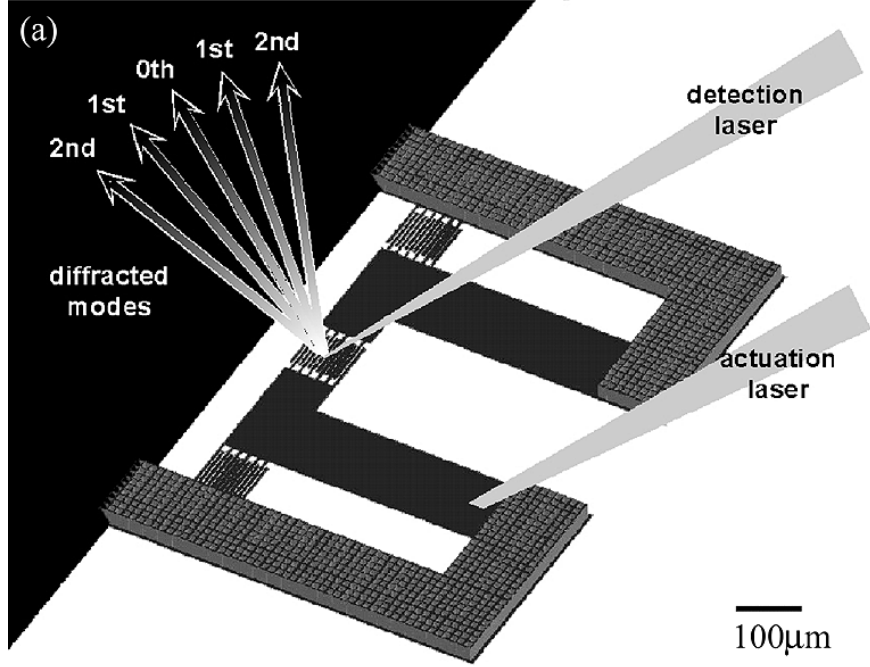

(b)

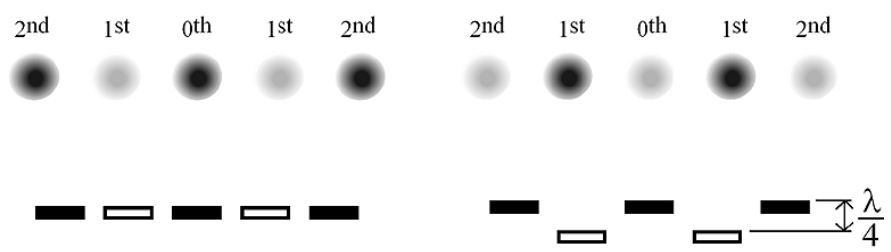

Fig. 1. (a) Device architecture, optical detection, and actuation schematic. (b) Pattern of diffraction modes and their dependence on the relative position of the interdigitated finger sets.

adjacent silicon nitride cantilevers $(500 \mu \mathrm{m} \times 100 \mu \mathrm{m} \times 1 \mu \mathrm{m})$ connected to the substrate with L-shaped silicon nitride stiff supports. The ID fingers at the termini of the cantilevers enable deflection sensing. Each cantilever has a thin layer of gold on one surface to enable covalent immobilization of bio-molecules [3], [5], [7], [9], [10]. Furthermore, the difference in thermal expansion coefficients of gold and silicon nitride causes a bimorph effect, which enables actuation of the cantilevers by heating.

The ID fingers between the two cantilevers are used to detect the differential bending, whereas the ID fingers between the cantilevers and the supports enable detection of the absolute bending of each cantilever. When the fingers are illuminated with a laser, the reflected light produces a diffraction pattern composed of several modes [see Fig. 1(b)]. The intensities of these modes depend on the relative out-of-plane distance between the ID finger sets. When the two finger sets are perfectly aligned, the even numbered modes have maximum intensity, whereas the odd numbered modes are dim. The opposite occurs when the distance between the two sets is an odd multiple of a quarter of the illumination wavelength $(\lambda / 4)$. An actuation laser allows the position of a single cantilever to be controlled, or biased, by locally heating its base and causing it to bend due to the bimorph effect.

Fig. 2 shows scanning electron micrographs of the structure. The L-shaped supports have a grid structure that was formed simultaneously with the flexible cantilevers. The grid structure is four times thicker than the $1-\mu \mathrm{m}$-thick flexible cantilevers [see Fig. 2(b) and (c)]. This ensures minimal bending of the support structure.
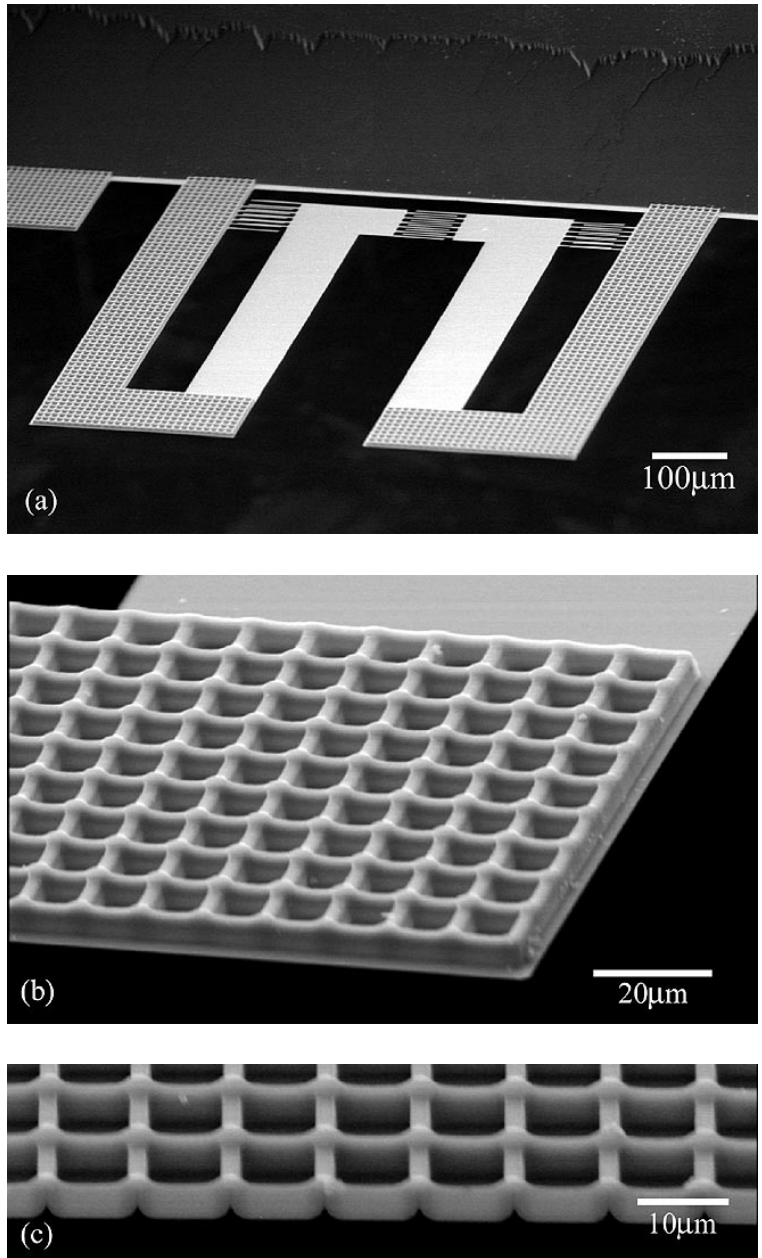

Fig. 2. Scanning electron micrographs of the sensor. (a) Entire sensor. (b) Support structure-cantilever interface. (c) Cross section of support structure.

Fig. 3(a) illustrates the main steps of the fabrication process. First, the grid pattern of the support structures was defined using photolithography on a four-inch, 500- $\mu$ m-thick $\langle 100\rangle$ silicon wafer. Then, $4 \mu \mathrm{m}$ deep trenches were formed by deep reactive ion etching (DRIE) of silicon in a time-multiplexed $\mathrm{SF}_{6} / \mathrm{C}_{4} \mathrm{~F}_{8}$ plasma for $4 \mathrm{~min}$. The photoresist was removed using a piranha clean (1: $3 \mathrm{H}_{2} \mathrm{O}_{2}-\mathrm{H}_{2} \mathrm{SO}_{4}$ ). In order to remove the passivation residue $\left(\mathrm{C}_{4} \mathrm{~F}_{8}\right)$ from the DRIE process, a thin layer of oxide was grown [not shown in Fig. 3(a)] at $1100{ }^{\circ} \mathrm{C}$ for 30 min, and then completely stripped using a buffered oxide etch. Next, the wafer was coated conformally with $1 \mu \mathrm{m}$ low stress silicon-rich (10:1) silicon nitride using low-pressure chemical vapor deposition to form the 4- $\mu \mathrm{m}$-thick support structure and the $1-\mu \mathrm{m}$-thick cantilever material simultaneously. The individual dies were defined by backside photolithography, followed by a plasma etch of the nitride in $\mathrm{CF}_{4}$. Again, the photoresist was removed using a piranha clean. The last photolithography step was done to pattern the devices on the front side which was followed by the plasma etch of the nitride in $\mathrm{CF}_{4}$. After removing the photoresist by using piranha, the silicon-nitride devices were released with a wet etch of the bulk silicon. The etch was carried out in a $\mathrm{KOH}$ solution ( $25 \%$ by volume) at $65^{\circ} \mathrm{C}$ over a period of $17 \mathrm{~h}$. Prior to gold deposition, a single device was cleaned using pi- 
(a)
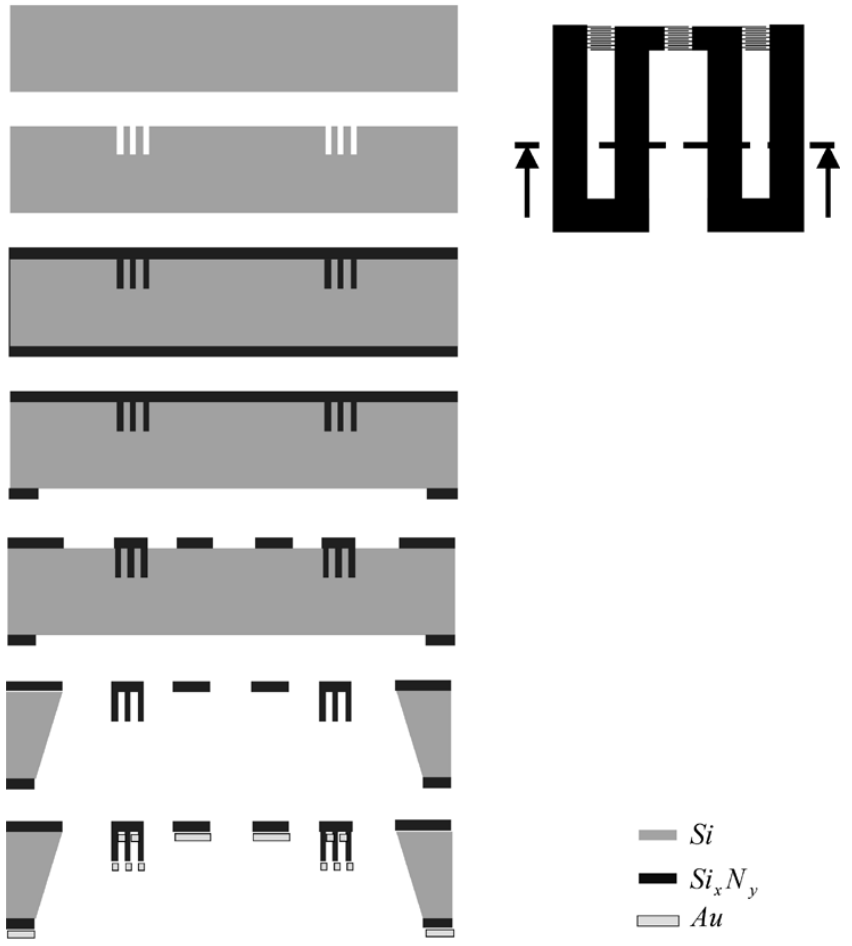

Si

$-S i_{x} N_{y}$ $\square A u$

(b)

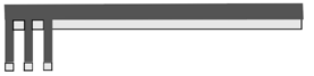

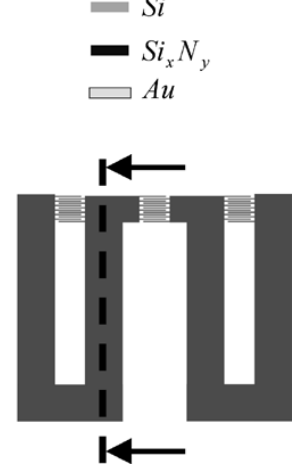

Fig. 3. Fabrication process. (a) Device cross section after each major fabrication step. From top to bottom: RCA cleaned $500 \mu \mathrm{m}\langle 100\rangle$ silicon wafer, DRIE formed trenches for support, low stress silicon-nitride deposition, backside patterning (photolithography and plasma etch) of silicon nitride, front side patterning (photolithography and plasma etch) of silicon nitride, Release with $\mathrm{KOH}$ etch of bulk silicon, e-beam deposition of gold. (b) Cross section of cantilever-support interface after gold deposition.

ranha $\left(1: 1 \mathrm{H}_{2} \mathrm{O}_{2}-\mathrm{H}_{2} \mathrm{SO}_{4}\right)$ in order to remove organics. Finally, $200 \AA$ of gold (with a $10 \AA \mathrm{Ti}$ adhesion layer) was deposited by electron-beam evaporation onto the bottom surface of the device. Fig. 3(b) shows a schematic of the completed cantilever cross section.

The overall geometry of the device enables simple fluidic delivery with commercially available pipettes. Fig. 4 shows insertion of a cantilever into a pipette. This method allows chemical functionalization of each cantilever surface individually, facilitating the device's use as a bio/chemical sensor.

\section{DeVICE CHARACTERIZATION}

The devices were tested by detecting the absolute and relative bending of the cantilevers in response to temperature changes. First, calibration was performed by measuring the intensity of a diffraction mode of the differential ID finger set. A 670-nm-wavelength laser diode (Hitachi HL6501MG) was used for actuation, and its power output was gradually
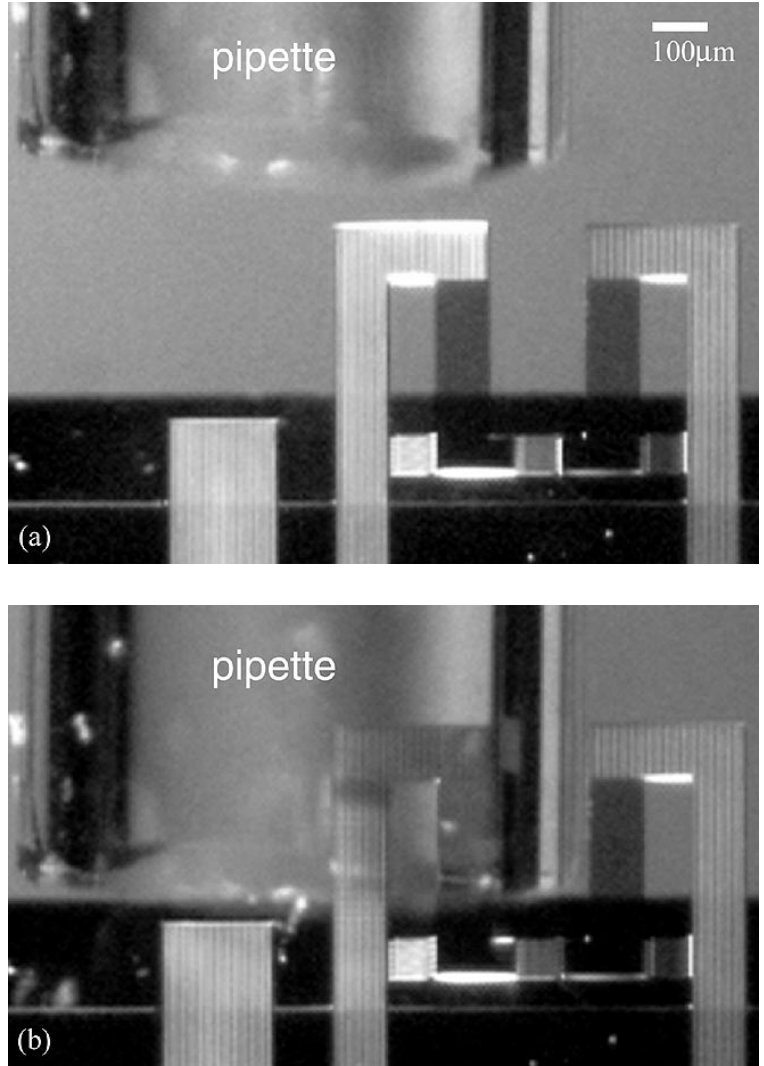

Fig. 4. Insertion of a cantilever into a commercially available glass pipette. (a) Before insertion. (b) After insertion.

increased from 1.5 to $18 \mathrm{~mW}$ to cause bending. The diffraction mode intensity was measured with a $3 \mathrm{~mW}, 635 \mathrm{~nm}$ wavelength laser diode (Sanyo DL3148-021) and a standard silicon photodiode (Thorlabs FDS100) through a notch filter. The output of the photodiode was converted to voltage using a current amplifier (Keithley 428). Data acquisition was carried out using a National Instruments Labview interface.

Fig. 5 shows the measured first diffraction mode intensity as a function of the relative deflection between the two cantilevers caused by heating one of the cantilevers. Also shown is the theoretical dependence of the first diffraction mode on relative deflection as predicted by (1) [11]

$$
I_{1} \propto \sin ^{2}\left(\frac{2 \pi}{\lambda} \xi\right)
$$

Here, $\xi$ represents the relative displacement between the two cantilevers, and $\lambda$ the illumination wavelength $(635 \mathrm{~nm})$. Deflection in nanometers was calibrated by using (1) and setting the distance between a minimum and a maximum to $\lambda / 4$. Since the intensity is periodic in $\xi$ and the initial distance between the two cantilevers is not known, the zero point of the abscissa in Fig. 5 was set arbitrarily. We suspect that the deviation from theoretical behavior at low deflections results from nonlinearities exhibited by the laser source when operated at low power.

The optimum operation point is the point of maximum slope of either curve shown in Fig. 5 ( 120 nm). At this bias point, the intensity of the diffraction mode is most sensitive to small changes in the relative distance of the ID finger sets. Most reported cantilever deflections resulting from biological reactions 


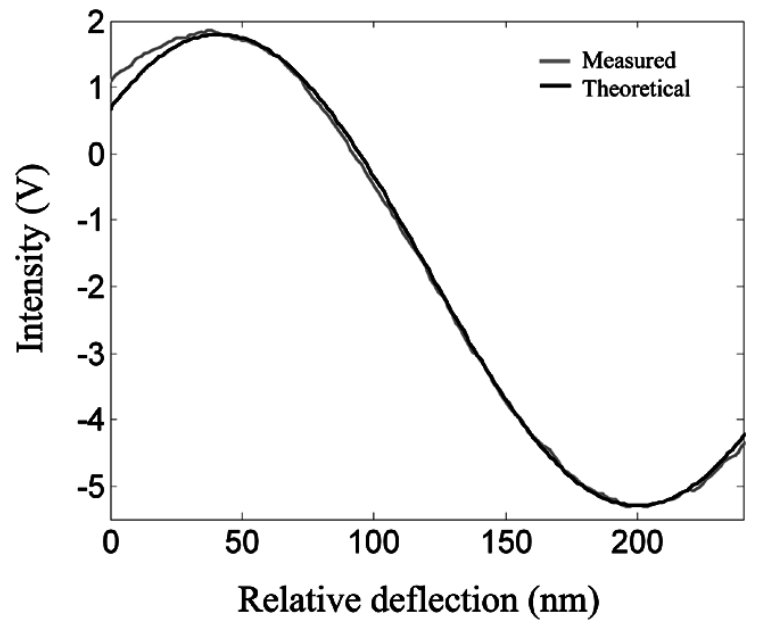

Fig. 5. Differential bending response in water as the actuation laser gradually heats one of the cantilevers. Measured is the first diffraction mode intensity detected by the photodiode and Theoretical is the behavior predicted by (1).

are much smaller than the maximum deflection shown in Fig. 5. For instance, Fritz et al. report a differential deflection caused by DNA hybridization to be $\sim 10 \mathrm{~nm}[7]$.

For an ideal differential sensor, the two adjacent cantilevers should be identical. However, slight changes in cantilever length during photolithography, or nonuniformities that occur during the deposition of the nitride and the gold could cause variations in the mechanical properties of the two cantilevers. We investigated the similarity of the two cantilevers by measuring their resonance frequencies. We measured the resonance frequency of each cantilever by modulating the actuation laser with a sine wave and gradually varying the frequency. The measurement was done in air to achieve large deflection at resonance. The deflection was measured with the absolute ID fingers. Fig. 6 shows that the resonance frequencies of the two cantilevers $(\sim 4.4 \mathrm{kHz})$ differ by only $85 \mathrm{~Hz}$, which corresponds to a variation of about $2 \%$. This is in good agreement with results shown in literature [7], and shows that the device can be used as a differential sensor.

To assess the effectiveness of differential sensing, we compared the differential and the absolute cantilever bending in response to ambient temperature changes, in the absence of the actuation laser. The device was placed in a closed 5-mL fluidic cell machined out of acrylic. Cold water $\left(4{ }^{\circ} \mathrm{C}\right)$ was injected into the cell. As the injected water warmed up to room temperature $\left(21^{\circ} \mathrm{C}\right)$, the bending of a single cantilever was detected using the absolute ID fingers. The experiment was immediately repeated with the differential ID fingers to measure the differential bending. Fig. 7 shows the absolute and the differential cantilever bending as a function of time as the injected water warms up to room temperature. Since the injected water warms up exponentially in time, the distance between the peaks increases.

From Fig. 7, it is clear that the differential measurement is much less affected by temperature changes when compared with the absolute one. Since both cantilevers have the same thermal response, detecting the differential bending can easily reduce the effect of temperature fluctuations, and provide a more reliable detection of specific bending caused by a bio/chemical

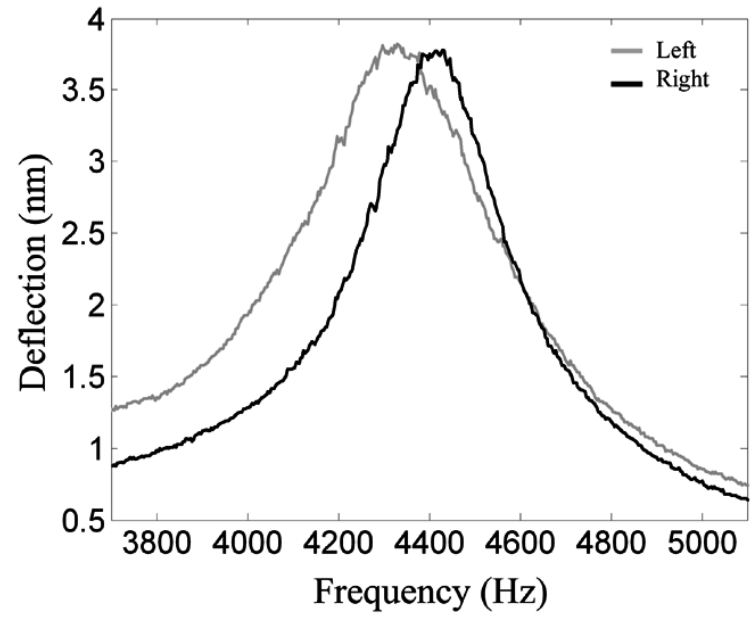

Fig. 6. RMS vibration amplitude of each cantilever versus the drive frequency of the actuation laser. RMS amplitudes were obtained sequentially in air by measuring the first diffraction mode intensity from the absolute interdigitated finger sets.

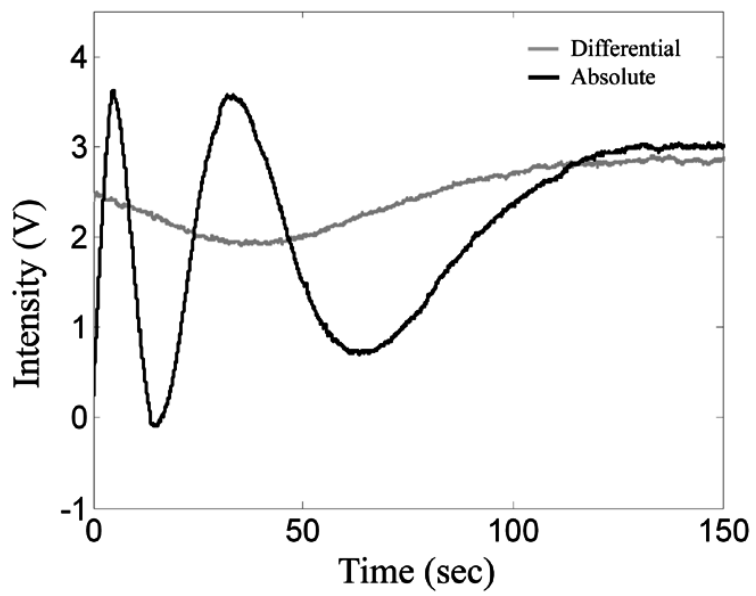

Fig. 7. Absolute deflection of one cantilever and differential deflection between two cantilevers in water, in response to ambient temperature change. At time $t=0$, cold water $\left(4^{\circ} \mathrm{C}\right)$ was injected into a 5 -mL fluidic cell, and allowed to warm up to room temperature $\left(21^{\circ} \mathrm{C}\right)$.

reaction. We estimate from the maxima of the slopes of the two curves that the differential measurement is approximately 50 times less sensitive to temperature changes than the absolute measurement. The temperature sensitivity of a cantilever with the material and geometric properties reported here is calculated as $70 \mathrm{~nm} /{ }^{\circ} \mathrm{C}[12]$. Accordingly, the differential thermal sensitivity is calculated as $1.4 \mathrm{~nm} /{ }^{\circ} \mathrm{C}$. In a system using a single cantilever, bending induced by a $1{ }^{\circ} \mathrm{C}$ temperature change could easily exceed that caused by a typical biological reaction.

Finally, we measured the sensor response to background noise (in the absence of the actuation laser). Fig. 8 shows an overlay of the measured spectrum in air and the magnitude of the frequency response of an ideal second-order harmonic oscillator predicted by (2) [13]

$$
X(\omega)=\frac{\sqrt{\frac{4 K_{b} T}{K \omega_{n}}}}{\sqrt{Q\left(1-\frac{\omega^{2}}{\omega_{n}^{2}}\right)^{2}+\frac{\omega^{2}}{Q \omega_{n}^{2}}}} .
$$




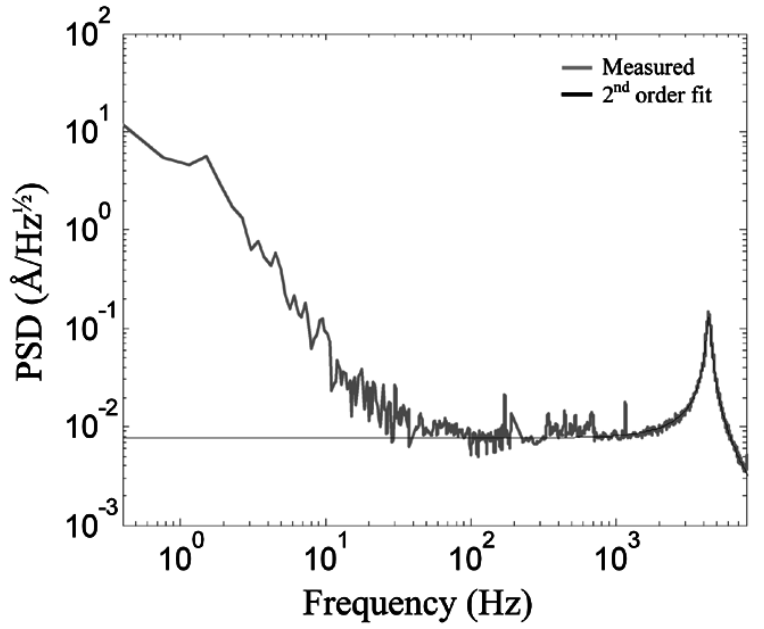

Fig. 8. Power spectral density of the differential cantilever deflection in air (Measured). Second-order fit is the frequency response of an ideal harmonic oscillator that has the same natural frequency, quality factor, and stiffness as a cantilever.

Here, $Q, K$, and $\omega_{n}$ represent the quality factor, stiffness and the fundamental frequency of the cantilever respectively. $K_{b}$, $T, \omega$, stand for the Boltzmann constant, room temperature, and excitation frequency, respectively. Equation (2) assumes that the low-frequency response of the cantilever is limited only by its thermomechanical noise level predicted by (3) [13]

$$
\delta=\sqrt{\frac{4 K_{b} T}{Q K \omega_{n}}} .
$$

The measured spectrum, as illustrated in Fig. 8 has flicker noise combined with second-order dynamics. Fitting the second-order dynamics of the measured spectrum to (2) for $\omega_{n}=(2 \pi) 4375 \mathrm{rad} / \mathrm{s}$ reveals: $Q=16.75$ and $K=0.061 \mathrm{~N} / \mathrm{m}$. The theoretical stiffness of the cantilever based on dimensions and material properties is $K=0.054 \mathrm{~N} / \mathrm{m}$ [14]. The difference between the two stiffness values could result either from the deviation of the actual material properties from the tabulated ones [15] or from variations in cantilever length and thickness during fabrication. The fit of (2) shows that the measured response over the frequency range $40-1000 \mathrm{~Hz}$ is the thermomechanical noise level of the cantilever $\left(\sim 0.008 \AA / \mathrm{Hz}^{1 / 2}\right)$. Hence, for applications in this frequency range, only the thermomechanical noise limits the sensitivity. For frequencies lower than $40 \mathrm{~Hz}$, resolution is dictated by flicker noise. Integration of the measured frequency spectrum in Fig. 8 and several others measured in air and in water (data not shown), over the range 0.4-40 Hz, reveals an rms noise level of $1 \mathrm{~nm}$, which compares well with the rms noise level of the response in Fig. $7(\sim 1 \mathrm{~nm})$. This noise floor is at least an order of magnitude less than many reported bending values [5], [7], [9] caused by various bio/chemical reactions.

\section{CONCLUSION}

We demonstrated the fabrication and characterization of a cantilever-based interferometric sensor that enables direct detection of differential deflection. Interferometric nature of the detection allows calibration of the displacement response only by knowing the illumination wavelength. The structure allows simple fluidic delivery onto cantilevers for chemical functionalization. The device was characterized by measuring the absolute and the differential cantilever bending induced by the bimorph effect, in response to both localized and ambient temperature changes. The similarity between the two adjacent cantilevers is well suited to enable the use of the device as a differential sensor. We showed that the differential sensing significantly reduces the effect of temperature changes in the background, enabling accurate measurement of specific sensor deflection. We anticipate that this sensor will be useful for investigating chemical and biological interactions that occur on cantilever surfaces, as well as photothermal spectroscopy.

\section{ACKNOWLEDGMENT}

The authors would like to thank K. Broderick of the MIT Microsystems Technology Laboratories where the device was fabricated. E. B. Cooper, N. Loh, and L. Arana provided significant help with fabrication and optical setup.

\section{REFERENCES}

[1] R. Berger, E. Delamarche, H. P. Lang, C. Gerber, J. K. Gimzewski, E. Meyer, and H.-J. Güntherodt, "Surface stress in the self-assembly of alkanethiols on gold," Science, vol. 276, pp. 2021-2023, June 1997.

[2] H. J. Butt, "A sensitive method to measure changes in the surface stress of solids," J. Colloid Interface Sci., vol. 180, pp. 251-260, 1996.

[3] G. Wu, H. Ji, K. Hansen, T. Thundat, R. Datar, R. Cote, M. Hagan, A. Chakraborty, and A. Majumdar, "Origin of nanomechanical cantilever motion generated from biomolecular interactions," PNAS, vol. 98, pp. 1560-1564, Feb. 2001.

[4] G. Y. Chen, T. Thundat, E. A. Wachter, and R. J. Warmack, "Adsorption-induced surface stress and its effects on resonance frequency of microcantilevers," J. Appl. Phys., vol. 77, pp. 3618-3623, Apr. 1995.

[5] G. Wu, R. H. Datar, K. M. Hansen, T. Thundat, R. J. Cote, and A. Majumdar, "Bioassay of prostate-specific antigen (PSA) using microcantilevers," Nature Biotech., vol. 19, pp. 856-860, Sept. 2001.

[6] A. M. Moulin, S. J. O'Shea, R. A. Bradley, P. Doyle, and M. E. Welland, "Measuring surface induced conformational changes in proteins," Langmuir, vol. 15, pp. 8776-8779, Sept. 1999.

[7] J. Fritz, M. K. Baller, H. P. Lang, H. Rothuizen, P. Vettiger, E. Meyer, H.-J. Güntherodt, C. Gerber, and J. K. Gimzewski, "Translating biomolecular recognition into nanomechanics," Science, vol. 288, pp. 316-318, Apr. 2000.

[8] S. R. Manalis, S. C. Minne, A. Atalar, and C. F. Quate, "Interdigital cantilevers for atomic force microscopy," Appl. Phys. Lett., vol. 69, pp. 3944-3946, Dec. 1996.

[9] J. Fritz, M. K. Baller, H. P. Lang, T. Strunz, E. Meyer, H.-J. Güntherodt, E. Delamarche, C. Gerber, and J. K. Gimzewski, "Stress at the solid-liquid interface of self-assembled monolayers on gold investigated with a nanomechanical sensor," Langmuir, vol. 16, pp. 9694-9696, Nov. 2000.

[10] T. Thundat, E. Finot, Z. Hu, R. H. Ritchie, G. Wu, and A. Majumdar, "Chemical sensing in fourier space," Appl. Phys. Lett., vol. 77, pp. 4061-4063, Dec. 2000.

[11] G. G. Yaralioglu, A. Atalar, S. R. Manalis, and C. F. Quate, "Analysis and design of an interdigital cantilever as a displacement sensor," $J$. Appl. Phys., vol. 83, pp. 7405-7415, June 1998.

[12] R. Barnes, R. J. Stephenson, C. N. Woodburn, S. J. O'Shea, M. E. Welland, T. Rayment, J. K. Gimzewski, and C. Gerber, "A femtojoule calorimeter using micromechanical sensors," Rev. Sci. Instrum., vol. 65, no. 12, pp. 3793-3798, Dec. 1994.

[13] D. Sarid, Scanning Force Microscopy. New York: Oxford Univ. Press, 1994, pp. 47-48.

[14] W. T. Thomson and M. D. Dahleh, Theory of Vibration With Applications, 5th ed. Englewood Cliffs, NJ: Prentice-Hall, 1998, p. 273.

[15] S. D. Senturia, Microsystem Design. Norwell, MA: Kluwer Academic, 2000, p. 196. 
Cagri A. Savran received the B.S. degree in mechanical engineering from Purdue University, West Lafayette, IN, in 1998 and the S.M. degree in mechanical engineering from the Massachusetts Institute of Technology (MIT), Cambridge, in 2000. His S.M. study concentrated on control systems and their applications to smart structures technology. He is currently working toward the Ph.D. degree at MIT, where his doctoral work involves microfabricated biosensors for label-free protein, and DNA detection.

Andrew W. Sparks received the S.B. degree in materials science and engineering from the Massachusetts Institute of Technology (MIT), Cambridge, in 1999.

He spent two summers at the Nippon Telegraph and Telephone Corporation,Tokyo, Japan, working in the area of holographic data storage. Since then, he has been pursuing the Ph.D. degree at the Massachusetts Institute of Technology (MIT), Cambridge, in the research group of Professor Manalis as a National Defense Science and Engineering Graduate Fellow.

His research interests include the design and microfabrication of unique scanning probes and the development of new nanoscale sensors.

Joachim Sihler received the Dipl.-Ing. degree in mechanical engineering from the University of Stuttgart, Stuttgart, Germany. He is currently pursuing the Ph.D. degree at the Massachusetts Institute of Technology (MIT), Cambridge.

His research interests include precision machine design and microelectromechanical systems.

Jian $\mathbf{L i}$ received the B.A. and M.S. degrees in precision instruments from Tsinghua University, Beijing, China, in 1997 and 2000, respectively. He is currently pursuing the Ph.D. degree with the Department of Mechanical Engineering, Massachusetts Institute of Technology (MIT), Cambridge.

His research interests are on the design, fabrication, and characterization of MEMS devices.

Wan-Chen Wu received the B.S. degree in mechanical engineering from National Taiwan University, Taipei, Taiwan, in 1996 and the M.S. degree in mechanical engeering from the Massachusetts Institute of Technology (MIT), Cambridge, in 1999. She is currently working toward the Ph.D. degree.

Dean E. Berlin was born in 1978 in London, England. He studied materials science and engineering with a focus on semiconductor device engineering at the Massachusetts Institute of Technology (MIT), Cambridge. He received the S.B. and S.M. degrees in 2000 and 2002, respectively.

At MIT, his final project was a joint collaboration with Applied Materials, Inc. involving the fabrication of silicon germanium waveguides by low pressure chemical vapor deposition. In 2002, he joined Applied Materials, Inc. and is currently in the Santa Clara, CA, headquarters developing and improving semiconductor processing equipment.
Thomas P. Burg (S'00) received the dipl.-phys. degree in physics from the Swiss Federal Institute of Technology (ETH) Zurich, Switzerland, in 2001. His thesis project at the Physical Electronics Laboratory at ETH involved the recognition of volatile organic compounds with CMOS-based capacitive microsensors.

He is currently working toward the Ph.D. degree in electrical engineering and computer science at the Massachusetts Institute of Technology (MIT), Cambridge. His research focuses on new methods for the label-free detection of biomolecules using microfabricated devices. Mr. Burg is supported by an MIT Presidential Fellowship.

Jürgen Fritz received the $\mathrm{Ph} . \mathrm{D}$. degree in experimental physics from the University of Basel, Switzerland, in 1998, working in a research laboratory of Novartis on biological applications of AFM.

After a postdoctoral study at the IBM Zurich Research Laboratory on nanomechanical sensors, he joined the Massachusetts Institute of Technology (MIT) Media Laboratory, Cambridge, in 2000, where he now works as a Research Scientist. His current research interests cover the fields of silicon biosensors, biophysics, and nanotechnology.

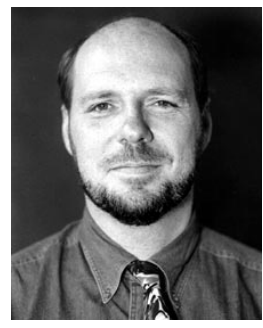

Martin A. Schmidt (S'88-M'85-SM'00) received the B.S. degree in electrical and computer engineering from Rensselaer Polytechnic Institute, Troy, NY, in 1981, and the S.M. and Ph.D. degrees in electrical engineering and computer science from the Massachusetts Institute of Technology (MIT), Cambridge, in 1983 and 1988, respectively. His Ph.D. research concerned microsensors for measurement of turbulent boundary layers.

In 1988, he joined the faculty of the Electrical Engineering and Computer Science Department, MIT, where he is currently a Professor of electrical engineering. He is also the Director of the Microsystems Technology Laboratories, MIT. His research interests are in microfabrication technologies for realization of micromechanical and biological reactors, micromachined turbine engines, and microactuators. He has served on the program committees for many of the major meetings in the microelectromechanical systems (MEMS) field and currently serves on the International Steering Committee for Solid-State Sensors and Actuators.

Dr. Schmidt was the recipient of the National Science Foundation Presidential Young Investigator Award and the Ruth and Joel Spira Teaching Award presented by MIT.

Scott R. Manalis received the B.S. degree in physics from the University of California, Santa Barbara, in 1994 and the Ph.D. degree in applied physics from Stanford University, Palo Alto, CA, in 1998.

Currently, he is an Assistant Professor of Media Arts \& Sciences and Biological Engineering at the Massachusetts Institute of Technology (MIT). His research interests are on the development of nanofabrication technologies for building molecular-scale devices, the use of MEMS for novel detection schemes, and the application of such devices to biology.

Dr. Manalis was the recipient of the Presidential Early Career Award for Scientists and Engineers (PECASE) from the Department of Defense. 